\title{
ASTROMETRIC PLATE REDUCTION WITH ORTHOGONAL FUNCTIONS AND MILLIARCSECONDS ACCURACY IN DEEP PROPER MOTION SURVEYS
}

\author{
DEVENDRA OJHA AND OLIVIER BIENAYMÉ \\ Observatoire de Besançon, BP 1615, F-25010 Besançon, France
}

We have been doing a sample survey in UBV photometry and proper motions as part of an investigation of galactic structure and evolution. The 3 fields in the direction of galactic anticentre $\left(l=167^{\circ}, b=47^{\circ}\right)$, centre $\left(l=3^{\circ}, b=47^{\circ}\right)$ and antirotation $\left(l=278^{\circ}, b=47^{\circ}\right)$ have been surveyed. The high astrometric quality of the MAMA machine (CAI, Paris) gives access to micronic accuracy (leading to $<2$ mas per year) on proper motions with a 35 years time base. The kinematical distribution of $\mathrm{F}$ and G-type stars have been probed to distances up to $2.5 \mathrm{kpc}$ above the galactic plane. We have derived the constrain on the structural parameters of the thin and thick disk components of the Galaxy (Ojha et al. 1994abc) :

- The scale lengths of the thin and thick disks are found to be $2.6 \pm 0.1$ and $3.3 \pm 0.5 \mathrm{kpc}$, respectively. The density laws for stars with $3.5 \leq \mathrm{M}_{V} \leq 5$ as a function of distance above the plane follow a single exponential with scale height of $\sim 260 \mathrm{pc}$ (thin disk) and a second exponential with scale height of $\sim 800 \mathrm{pc}$ (thick disk) with a local normalization of $5-6 \%$ of the disk.

- The thin disk population was found with $(\langle\mathrm{U}+\mathrm{W}\rangle,\langle\mathrm{V}\rangle)=(1 \pm 4$, $-14 \pm 2) \mathrm{km} / \mathrm{s}$ and velocity dispersions $\left(\sigma_{U+W}, \sigma_{V}\right)=(35 \pm 2,30 \pm 1) \mathrm{km} / \mathrm{s}$. The thick disk population was found to have a rotational velocity of $\mathrm{V}_{\text {rot }}$ $=177 \mathrm{~km} / \mathrm{s}$ and velocity dispersions $\left(\sigma_{U}, \sigma_{V}, \sigma_{W}\right)=(67,51,42) \mathrm{km} / \mathrm{s}$. No dependence with $\mathrm{r}$ and $\mathrm{z}$ distances was found in the asymmetric drift measurements of the thick disk population.

\section{References}

Ojha D.K., Bienaymé O., Robin A.C., Mohan V., 1994a, A\&A 284, 810 Ojha D.K., Bienaymé O., Robin A.C., Mohan V., 1994b, A\&A 290, 771

Ojha D.K., 1994c, Ph.D. thesis, Observatoire de Strasbourg, France (in preparation) 